\title{
Relación neutrófilos/linfocitos y plaquetas/linfocitos y su correlación con los reactantes de fase aguda y la actividad de la enfermedad en pacientes con artritis reumatoide
}

\section{Neutrophil/lymphocyte and platelet/lymphocyte ratio and correlation with acute phase reactants and disease activity in patients with rheumatoid arthritis}

\author{
Mercedes Cecilia Córdoba ${ }^{1}$ (D) , Ana Maria Bertoli ${ }^{1}$ (D) , María José López Perez ${ }^{1}$, José Pablo Sironi ${ }^{1}$, \\ María Teresa Apaz ${ }^{1}$, Luis Eduardo Marcelo Sánchez Freytes ${ }^{1}$ \\ 1 Universidad Católica de Córdoba, Facultad de Ciencias de la Salud, Clínica Universitaria Reina Fabiola, Servicio de Reumatología. \\ Correspondencia: Mercedes Cecilia Córdoba. E-mail: cecordoba26@gmail.com
}

\section{Resumen}

INTRODUCCIÓN: La relación neutrófilos/linfocitos (RNL) y plaquetas/linfocitos son medidas sensibles de inflamación. El objetivo de este estudio fue correlacionar la RNL y RPL con VSG, PCR (Proteína C Reactiva) y el índice de actividad de la enfermedad DAS28- PCR (Medida de la actividad de la enfermedad DAS28-PCR), así como determinar los puntos de corte de la RNL y RPL indicativos de remisión de la enfermedad, actividad leve, moderada y grave determinada por DAS28-PCR, en pacientes con diagnóstico de artritis reumatoide.

MATERIALES Y METODOS: Estudio retrospectivo en el que se evaluaron pacientes adultos con diagnóstico de AR. La correlación de la RNL y RPL con VSG, PCR y el índice de actividad de la enfermedad DAS28-PCR se evaluó con test de Pearson. Los valores de corte de la RNL y RPL para discriminar actividad de la enfermedad (remisión, actividad baja, moderada y alta) se analizó con curvas ROC.

RESULTADOS: Evaluamos 151 mediciones correspondientes a 55 pacientes. La RNL tuvo una correlación débil con VSG ( $r=0.065)$, PCR ( $r=0.23)$ y DAS-28 PCR ( $r=0.18)$. La RPL tuvo una correlación débil con VSG ( $\mathrm{r}=0.22)$, moderada con PCR ( $\mathrm{r}=0.38)$ y débil con DAS-28 PCR ( $\mathrm{r}=0.20)$. El área bajo la curva de la RNL para discriminar remisión, actividad baja, moderada y grave fue de 0.61, 0.61, 0.58 y 0.35 , respectivamente y para la RPL de $0.55,0.55,0.45$ y 0.24 , respectivamente.

CONCLUSIÓN: En este estudio, la correlación entre RNL y RPL con reactantes de fase aguda y actividad de la enfermedad fue débil y la capacidad discriminativa para establecer el estado de actividad de la enfermedad fue baja. De acuerdo a estos resultados, estos indicadores no serían útiles para discriminar actividad de la enfermedad en pacientes con AR.

Palabras claves: Relación neutrófilos/linfocitos, relación plaquetas/linfocitos, artritis reumatoide, DAS28- PCR. 
Córdoba M.C, Bertoli A.M, López Pérez M.J, Sironi J.P, Apaz M.T, Sánchez Freytes L.E.M. Relación neutrófilos/linfocitos y plaquetas/linfocitos y su correlación con los reactantes de fase aguda y la actividad de la enfermedad en pacientes con artritis reumatoide.

\section{Abstract}

INTRODUCTION: The neutrophil/lymphocyte ratio (NLR) and the platelet/lymphocyte ratio (PLR) are well-recognized sensitive measures of inflammation in several diseases. The objectives of this study were to correlate the NLR and PLR with ESR, CRP (C Reactivy Protein) and disease activity as measured with the DAS28-CRP (Disease Activite Score-CRP) and to determine the cut-off points of the NLR and PLR indicative of remission, mild, moderate and severe activity.

MATERIALS AND METHODS: This is a retrospective study in which we included adult patients with diagnosis of RA. The correlation of NLR and PLR with ESR, CRP and the DAS28-CRP was evaluated with the Pearson's test. Cut-off values of the NLR and PLR to discriminate disease activity (remission, low, moderate and high disease activity) were analyzed with ROC curves using the DAS-28 CRP as the gold standard.

RESULTS: We included 151 measurements corresponding to 55 patients. The NLR had a weak correlation with ESR ( $\mathrm{r}=0.065), \mathrm{CRP}(\mathrm{r}=0.23)$ and DAS-28 CRP $(\mathrm{r}=0.18)$. The PLR had a weak correlation with ESR $(r=0.22)$, moderate with CRP $(r=0.38)$ and weak with the DAS-28 CRP $(r=0.20)$. The areas under curve of the NLR to discriminate remission, low, moderate and severe disease activity were $0.61,0.61,0.58$ and 0.35 , respectively, and for the PLR were $0.55,0.55,0.45$ and 0.24 , respectively.

CONCLUSION: In this study, we found a weak to moderate correlation of the RNL and RPL with acute phase reactants and disease activity. Likewise, the discriminative capacity of RNL and RPL was low to establish the disease activity status in this patient sample. Therefore, these indicators would not be suitable to address disease activity in patients with RA.

KeyWords: Neutrophil / lymphocyte ratio, platelet / lymphocyte ratio, rheumatoid arthritis, DAS28CRP.

\section{Introducción}

La artritis reumatoide (AR) es una enfermedad autoinmune, crónica, asociada con inflamación sistémica que afecta principalmente las articulaciones. La actividad de la enfermedad no controlada adecuadamente, inexorablemente conlleva a daño estructural articular, discapacidad y aumento de mortalidad ${ }^{1-3}$.

Actualmente, el objetivo del tratamiento es alcanzar la remisión clínica y con ello, reducir los síntomas y la progresión radiográfica. Para acompañar este paradigma, en las últimas dos décadas asistimos no solo al advenimiento de nuevas intervenciones terapéuticas, sino también a un monitoreo de la actividad de la enfermedad más estricto, apoyado por el desarrollo tecnológico (tal es el caso de las imágenes aplicadas a la evaluación diagnóstica y de la actividad de la enfermedad) y por la utilización de indicadores de actividad clínicos y de laboratorio ${ }^{4-5}$.

Los cambios en la relación de neutrófilos/linfocitos (RNL) y la relación de plaquetas/ linfocitos (RPL) obtenidos por la proporción de estas células entre sí, se han discutido en diferentes situaciones clínicas, incluyendo AR temprana y AR establecida en tratamiento con agentes biológicos ${ }^{6-9}$. Se ha hipotetizado que estas relaciones podrían utilizarse como equivalentes de otros reactantes de fase aguda, con un uso potencial como indicador de actividad y recaída de la enfermedad ${ }^{10-11}$. Por ende, estas relaciones podrían sustituir a los reactantes de fase aguda en caso de no estar disponibles, además de poseer una ventaja económica en comparación con la proteína $\mathrm{C}$ reactiva $(\mathrm{PCR})$.

\section{Objetivo}

Correlacionar la relación de neutrófilos/linfocitos (RNL) y la relación de plaquetas/ linfocitos (RPL) con la velocidad de sedimentación globular, PCR y el índice de actividad de la enfermedad DAS-28 PCR

Determinar los puntos de corte de la RNL y RPL indicativos de remisión de la enfermedad, actividad leve, moderada y grave establecida por DAS28- PCR.

\section{Material y métodos}

Se realizó un estudio retrospectivo, analítico, en el que se incluyeron pacientes adultos mayores de 18 años con diagnóstico de artritis reumatoide de acuerdo a los criterios diagnósticos del Colegio Americano de Reumatología / Liga Europea Contra el Reumatismo (ACR/EULAR) 2010 12 que fueron atendidos en la Clínica Universitaria Reina Fabiola entre los años 2018-2020.

Mediante la revisión de los registros de historias clínicas se recolectaron los siguientes datos:

a) Variables demográficas: edad y sexo al nacimiento. 
b) Variables clínicas: tiempo de evolución de la enfermedad y actividad de la enfermedad evaluada mediante el DAS28- PCR ${ }^{13}$, clasificándose como: remisión $(\leq 2.6)$, actividad baja $(>2.6-3.2)$, moderada $(>3.2-5.1)$ y grave $(>5.1)^{14}$.

c)Variables de laboratorio: seropositividad para factor reumatoideo y anticuerpos anti péptidocitrulinado, recuento de leucocitos (x 109/1), neutrófilos (x10 $/ 1)$, linfocitos $\left(\mathrm{x} 10^{9} / 1\right)$, plaquetas (x109/1), valor de PCR (mg/l), VSG (mm/1 ${ }^{\circ}$ hora), RNL y RPL (obtenida del cociente entre ambos valores).

d)Variables terapéuticas: uso al momento de la evaluación de antinflamatorios no esteroides (AINES), glucocorticoides, drogas modificadoras de la enfermedad sintéticas convencionales (DMARDsc), sintéticas dirigidas (DMARDsd) y biológicas (DMARDb).

Se excluyeron los datos de los pacientes que al momento de la evaluación presentaran una situación clínica que pudiera modificar la RNL, RPL o los reactantes de fase aguda (VSG y PCR): infección activa en las últimas dos semanas, insuficiencia renal crónica y presencia de neoplasias.

Análisis estadístico: Las características de la muestra de pacientes se reportó con estadística descriptiva. La correlación de la RNL y RPL con VSG, PCR y el índice de actividad de la enfermedad DAS28-PCR se realizó con test de correlación de Pearson. Los valores de corte de la RNL y RPL para discriminar actividad de la enfermedad: remisión, baja, moderada y alta se analizó con curvas ROC utilizando el DAS28PCR como prueba estándar de oro.

El estudio se realizó bajo las normas de la declaración de Helsinki, la ley nacional 25.326/00 de protección de los datos personales (Ley Hábeas Data) y la ley provincial 9694/09 del sistema de evaluación, registro y fiscalización de las investigaciones en salud. El estudio fue evaluado y aprobado por el área de investigación del Departamento de Docencia, Investigación y Compromiso Social de la Clínica Universitaria Reina Fabiola y por la Secretaría de Investigación de la Facultad de Ciencias de la Salud, Universidad Católica de Córdoba.

\section{Resultados}

El estudio incluyó 151 mediciones de reactantes de fase aguda (VSG y PCR), RNL y RPL así como también de DAS28-PCR correspondientes a 55 pacientes.

Los pacientes tenían una edad media (desviación estándar - DE) de 52.4 (14.35) años, siendo la mayoría de sexo femenino 45 (82\%). La mediana (rango intercuartilo-RIC) del tiempo de evolución de la enfermedad fue de 72 (120) meses. La mayoría de los pacientes eran seropositivos, tanto como para péptido cíclico citrulinado (62\%), como para factor reumatoide (84\%). La media (DE) del DAS28-PCR fue de 0.70 (0.98); 92 (61\%) pacientes estaban en remisión, 28 (18.5\%) con actividad moderada, 21 (14\%) actividad leve y 10 $(6.62 \%)$ presentaban actividad grave.

Las características de laboratorio de los pacientes se muestran en la (Tabla1). La mayoría de las pacientes estaban en tratamiento con antinflamatorios no esteroideos (58 \%), glucocorticoides (47 \%) y DMARD convencionales (91\%). Estos datos se muestran en la (Tabla 2).

Tabla 1. Características de laboratorio de pacientes con artritis reumatoide.

\begin{tabular}{lc}
\hline \multicolumn{1}{c}{ Laboratorio } & $\begin{array}{c}\text { TOTAL }=151 \\
\text { N(\%) o Media (DE) }\end{array}$ \\
\hline Hemoglobina (gr/dl) & $13.01(1.29)$ \\
Leucocitos (mm $\left.{ }^{3}\right)$ & $7128(2006)$ \\
Neutrófilos Absolutos (x 10 $/ \mathrm{L})$ & $4299(1667)$ \\
Linfocitos absolutos (x 10 $/ \mathrm{L})$ & $2094(704)$ \\
Plaquetas $\left(\mathrm{mm}^{3}\right)$ & $272.636(79.636)$ \\
VSG $(\mathrm{mm} / \mathrm{h})$ & $17.46(16.45)$ \\
PCR $(\mathrm{gr} / \mathrm{l})$ & $8.12(19.17)$ \\
RNL (\%) & $2.20(1.02)$ \\
RPL (\%) & $140.02(51.72)$ \\
CCP* & $45(82)$ \\
FR $\dagger$ & $48(87.2)$ \\
\hline
\end{tabular}

*Péptido cíclico citrulinado, factor reumatoide.

Tabla 2. Intervenciones terapéuticas de pacientes con artritis reumatoide

\begin{tabular}{lcc}
\hline \multicolumn{1}{c}{ Tratamiento } & \multicolumn{2}{c}{ TOTAL $=\mathbf{5 5}$} \\
& N & $\mathbf{( \% )}$ \\
\hline AINEs* & 32 & 58 \\
Glucocorticoides & 26 & 47.2 \\
DMARD $\dagger$ sintético convencional: & 50 & 91 \\
Metotrexate & 42 & 76.3 \\
Leflunomida & 5 & 9 \\
Hidroxicloroquina & 3 & 5.4 \\
DMARD sintético dirigido: & & \\
Baricitinib & 6 & 11 \\
DMARD biológico: & 18 & 33 \\
Anti TNF & 12 & 22 \\
Inhibidores IL-6 & 3 & 5.4 \\
Agonista CTLA-4 & 3 & 5.4 \\
\hline *antiinflamatorio no esteroideos. $\dagger$ droga modificadora de la enfermedad.
\end{tabular}


Córdoba M.C, Bertoli A.M, López Pérez M.J, Sironi J.P, Apaz M.T, Sánchez Freytes L.E.M. Relación neutrófilos/linfocitos y plaquetas/linfocitos y su correlación con los reactantes de fase aguda y la actividad de la enfermedad en pacientes con artritis reumatoide.

\section{Correlación entre RNL con reactantes de fase aguda (VSG y PCR) y DAS28- PCR.}

La correlación de la RNL con la VSG $(r=0.065$; $p=$ $0.430)$, PCR $(r=0.23 ; p=0.004)$ y con el DAS-28 PCR $(r=0.18 ; p=0.027)$ fueron débiles. Estos datos se muestran en las (Figura 1)
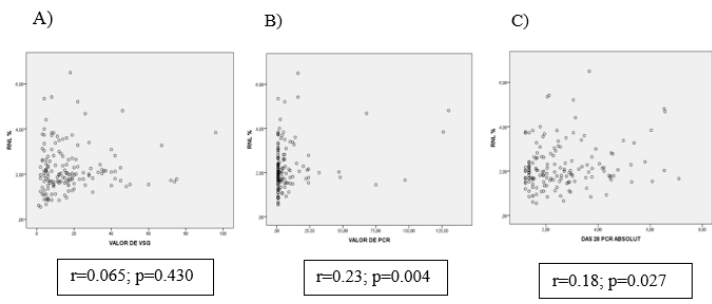

Figura 1. Correlación de Pearson entre relación neutrófilos/linfocitos y A) Eritrosedimentación, B) Proteína C reactiva, DAS28-VSG.

\section{Correlación entre RPL con reactantes de fase aguda (VSG y PCR) y DAS28-PCR.}

La correlación de la RPL con la VSG $(r=0.22 ; \mathrm{p}=$ $0.006)$ fue débil, con la PCR ( $r=0.38 ; \mathrm{p}=0.001)$ moderada y con el DAS28- PCR $(r=0.20 ; p=$ 0.016 ) débil. Esos datos se muestran en (la Figura 2).

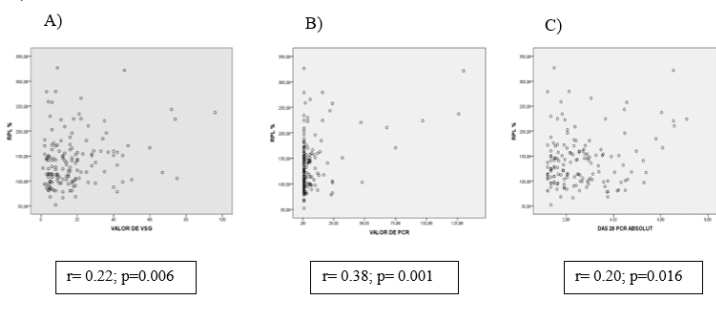

Figura 2. Correlación de Pearson entre la relación plaquetas/linfocitos y A) Eritrosedimentación, B) Proteína C reactiva, C) DAS28-PCR.

\section{Áreas bajo la curva de la RNL y RPL indicativos de remisión, actividad leve, moderada y grave de la enfermedad determinada por DAS28- PCR.}

El desempeño de la RNL para discriminar remisión y actividad baja de la enfermedad muestra un área bajo la curva similar (AUC) de $0.61(\mathrm{EE}=0.052$; IC 95\% 0.51-0.71), mientras que para actividad moderada presentó un AUC de 0.58 $(\mathrm{EE}=0.058$; IC 95\% 0.47-0.69) y para actividad grave una AUC de 0.35 (EE= 0.096; IC 95\% 0.170.54 ) tomando al DAS28-PCR como prueba de referencia. Estos resultados se muestran en la (Figura 3).

Por su parte, la RPL presentó una AUC para discriminar remisión y baja actividad de la enfermedad de 0.55 ( $\mathrm{EE}=0.61$; IC 95\% 0.43-0.66), para actividad moderada de la enfermedad presentó un AUC 0.45 (EE=0.067; IC 95\% 0.320.58 ), mientras que para actividad grave el AUC fue de $0.24 \quad(E E=0.082 ;$ IC $95 \% \quad 0.08-0.40)$ tomando como prueba de referencia al DAS28PCR. Estos datos se muestran en la (Figura 4).

Dado que los intervalos de confianza de las áreas bajo la curva fueron cercanos o cruzaron el 0.5 , no se justificó obtener valores de corte para discriminar los diferentes niveles de actividad de la enfermedad.
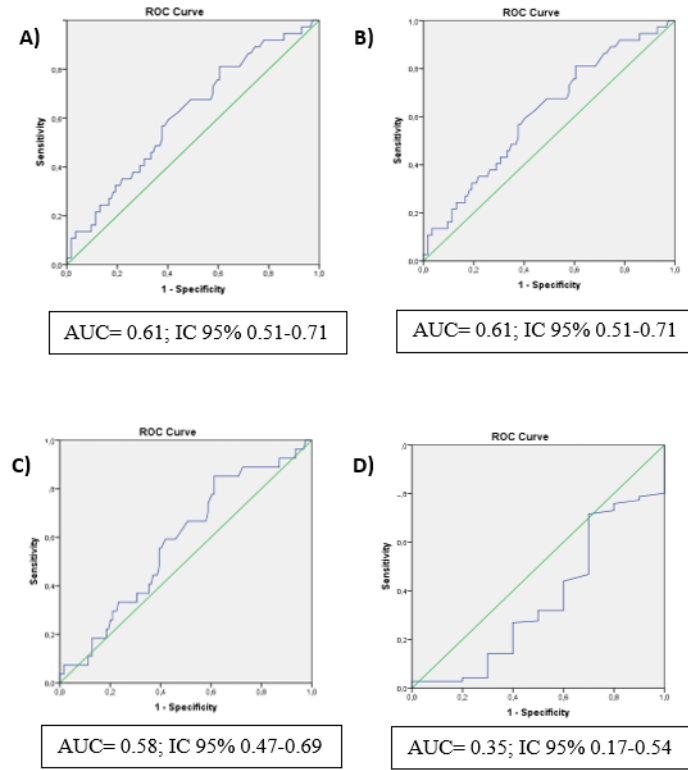

Figura 3. Curvas ROC para demostrar la capacidad discriminatoria de la relación neutrófilos/linfocitos y DAS28- PCR en: A)- remisión, B)- actividad leve, C)moderada y D)- grave de la enfermedad.
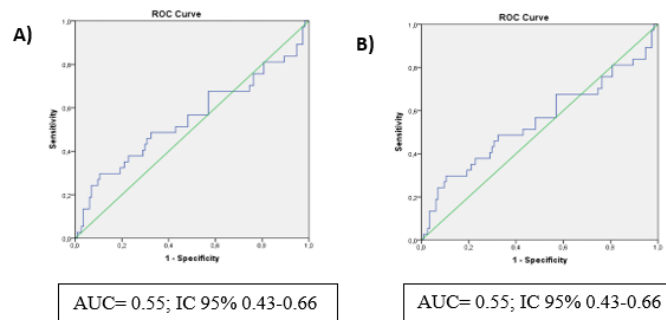

c)
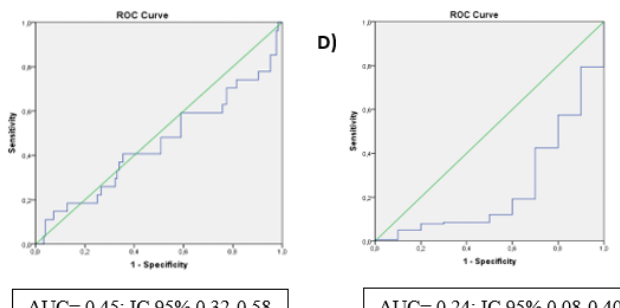

$\mathrm{AUC}=0.24 ;$ IC $95 \% 0.08-0.40$

Figura 4. Curvas ROC para demostrar la capacidad discriminatoria de la relación plaquetas/linfocitos y DAS28 -PCR en: A)- remisión, B)- actividad leve, C)moderada y D)- grave de la enfermedad.

\section{Discusión}

Los resultados de nuestro estudio muestran que tanto la RNL como la RPL tuvieron una 
correlación positiva, aunque débil a moderada con los reactantes de fase aguda y actividad de la enfermedad. Estos hallazgos son consistentes con lo publicado en otras cohortes de pacientes con artritis en los que, si bien se encontró una correlación positiva de ambos parámetros con indicadores de actividad de la enfermedad y reactantes de fase aguda, la misma fue, nuevamente, débil a moderada ${ }^{7,11,15-17}$.

La capacidad discriminativa de la RNL para diferenciar remisión y baja actividad de la enfermedad fue pobre en nuestro estudio; esto es consistente con lo publicado por Chandrashekara $\mathrm{S}$. et $\mathrm{al}^{17}$. Estos autores estimaron un AUC de 0.65 (CI 95\% 0.55-0.74) para discriminar remisión de la enfermedad por DAS28-PCR y un punto de corte de 1.4 para clasificar pacientes en remisión profunda con una alta especificidad $(90 \%)$ pero con una baja sensibilidad (24\%). Por su parte, la capacidad discriminativa entre RNL y RPL para valorar actividad moderada y grave de la enfermedad fueron bajos en nuestro estudio por lo que no determinamos valores de corte para clasificar a los pacientes en los diferentes niveles de actividad de la enfermedad ${ }^{18}$.

El presente estudio tiene algunas limitaciones. Es un estudio retrospectivo en el que la mayoría de los pacientes se encontraba en remisión clínica de la enfermedad. En los pacientes con AR en remisión es esperable encontrar niveles inconsistentes de reactantes de fase aguda ${ }^{18,19}$, especialmente de PCR, por lo que las correlaciones de ésta y del DAS28-PCR con la RNL y RPL podrían no ser óptimas. De hecho, este hallazgo, también fue reportado por Uslu y col ; en este estudio se observó una correlación menor entre el DAS28PCR y RNL y RPL en pacientes en remisión en comparación con los pacientes con enfermedad activa. También es posible, que algunos de los pacientes en remisión presentaran sinovitis subclínica no detectada por los reactantes de fase aguda ni por el DAS28-PCR, de modo que las correlaciones no fueran lo suficientemente fuertes. Por último, los valores estudiados corresponden a pacientes en tratamiento y como sabemos, algunas intervenciones terapéuticas tienen diferentes efectos sobre los parámetros hematológicos y reactantes de fase aguda.

\section{Conclusión}

En el presente estudio encontramos una correlación positiva pero débil entre RNL y RPL con reactantes de fase aguda y actividad de la enfermedad. Igualmente, la capacidad discriminativa de la RNL y RPL fue baja para establecer el estado de actividad de la enfermedad en este grupo de pacientes. A pesar que esos índices han sido propuestos como subrogados de inflamación adecuados para monitorear actividad de la enfermedad en AR, nuestro estudio, en base a los resultados obtenidos, no da soporte a la utilidad de la RNL y RPL en la evaluación de pacientes con AR, esto podría corresponderse con estudio retrospectivo con un número escaso de pacientes.

\section{Bibliografía}

1. Smolen JS, Aletaha D, McInnes IB. Rheumatoid arthritis. Lancet. 2016; 388:2023?2038.

2. Tobón GJ, Youinou P, Saraux A. The environment, geo?epidemiology, and autoimmune disease: rheumatoid arthritis. J Autoimmun. 2010; 35:10?14.

3. Wolfe F, Cathey MA. The assessment and prediction of functional disability in rheumatoid arthritis. J Rheumatol. 1991; 18:1774.

4. Singh JA, Saag KG, Bridges SL Jr, et al. 2015 American College of Rheumatology Guideline for the Treatment of Rheumatoid Arthritis. Arthritis Care Res (Hoboken) 2016; 68:1?25.

5. Singh JA, Furst DE, Bharat A, et al. 2012 update of the 2008 American College of Rheumatology recommendations for the use of disease?modifying antirheumatic drugs and biologic agents in the treatment of rheumatoid arthritis. Arthritis Care Res (Hoboken). 2012; 64:625?639.

6. Koiwa M, Goto S, Takahashi K, Kamada T, Takai S, Nakamura H. Neutrophil/lymphocyte ratio in patients with rheumatoid arthritis treated with biological agents. J Nippon Med Sch. 2016; 83:118?124.

7. Peng YF, Cao L, Zeng YH, et al. Platelet to lymphocyte ratio and neutrophil to lymphocyte ratio in patients with rheumatoid arthritis. Open Med (Wars). 2015; 10:249?253

8. Ghang B, Kwon O, Hong S, Lee CK, Yoo B, Kim YG. Neutrophil?tolymphocyte ratio is a reliable marker of treatment response in rheumatoid arthritis patients during tocilizumab therapy. Mod Rheumatol. 2017; 27:405?410.

9. Zengin O, Onder ME, Kalem A, et al. New inflammatory markers in early rheumatoid arthritis. Z Rheumatol. 2018; 77:144?150. 
10. Chandrashekara S, Rajendran A, Jaganath AB, Krishnamurthy R. Neutrophillymphocyte ratio, pain perception, and disease activity score may serve as important predictive markers for sustained remission in rheumatoid arthritis. Reumatismo 2015; 67: 109-15.

11. Uslu AU, Kucuk A, Sahin A et al. Two new inflammatory markers associated with Disease Activity Score-28 in patients with rheumatoid arthritis: neutrophil-lymphocyte ratio and platelet-lymphocyte ratio. Int $\mathbf{J}$ Rheum Dis 2015; 18: 731-5.

12. Aletaha D, Neogi T, Silman AJ et al. Rheumatoid arthritis classification criteria: An American College of Rheumatology/European League Against Rheumatism collaborative initiative. Ann Rheum Dis, 2010; 69(9): 1580-88.

13. Prevoo ML, van't Hof MA, Kuper HH, et al. Modified disease activity scores that include twenty-eight-joint counts. Development and validation in a prospective longitudinal study of patients with rheumatoid arthritis. Arthritis Rheum 1995; 38 (1):44-48.

14. Van Tuyl LH, Lems WF, Boers M: Measurement of stiffness in patients with rheumatoid arthritis in low disease activity or remission: a systematic review. BMC Musculoskelet Disord 2014; 15, 28.

15. Tekeoglu I, Gurol G, Harman H, Karakece E, Ciftci I. Overlooked hematological markers of disease activity in rheumatoid arthritis. International Journal of Rheumatic Diseases 2015.
16. Ridvan M, Berivan B, Abdurrahman T, Utku B, Nuh A, Mehmet A, Seminur H, Berna G. The Association between Neutrophil/Lymphocyte Ratio and Disease Activity in Rheumatoid Arthritis and Ankylosing Spondylitis. Journal of Clinical Laboratory Analysis 30: (2016).

17. Chandrashekara S, Mukhtar Ahmad M, Renuka P, Anupama K. Characterization of neutrophil-to-lymphocyte ratio as a measure of inflammation in rheumatoid arthritis. International Journal of Rheumatic Diseases 2017; 20: 1457-1467.

18. Cerda J, Cifuentes L. Uso de curvas ROC en investigación clínica: Aspectos teóricoprácticos. Rev. chil. infectol. 2012; 29(2): 138-141.

19. Asmussen R, Antonsen S, J. Hansen IMAB0190 The influence of variation in creactive protein values on the das 28 scoreAnnals of the Rheumatic Diseases 2013;72: A844.

20. Chandrashekara, S. and Priyanka, B.U. (2013), Remission in rheumatoid arthritis by different criteria does not converge over the inflammatory markers. Int J Rheum Dis, 16: 291-296.

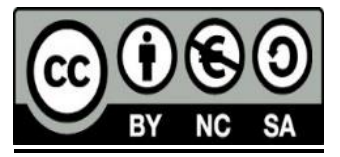

